\title{
Measurement of the $\mathrm{e}^{+} \mathrm{e}^{-} \rightarrow \mathrm{b} \overline{\mathrm{b}}$ and $\mathrm{e}^{+} \mathrm{e}^{-} \rightarrow \mathrm{c} \overline{\mathrm{c}}$ forward-backward asymmetries at the $Z^{0}$ resonance
}

\section{L3 Collaboration}

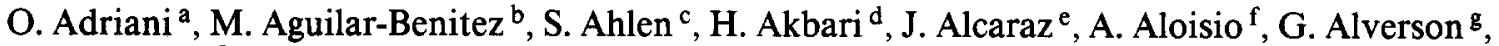
M.G. Alviggi ${ }^{f}$, G. Ambrosi ${ }^{\mathrm{h}}$, Q. An ${ }^{\mathrm{i}}$, H. Anderhub ${ }^{\mathrm{j}}$, A.L. Anderson ${ }^{\mathrm{k}}$, V.P. Andreev ${ }^{\ell}$, T. Angelov ${ }^{k}$, L. Antonov ${ }^{\text {m }}$, D. Antreasyan ${ }^{n}$, P. Arce $^{\text {b }}$, A. Arefiev ${ }^{\circ}$, A. Atamanchuk ${ }^{\ell}$, T. Azemoon ${ }^{\text {p }}$, T. Aziz ${ }^{\text {q,r }}$, P.V.K.S. Baba ${ }^{i}$, P. Bagnaia ${ }^{\text {s }}$, J.A. Bakken ${ }^{t}$, L. Baksay ${ }^{\text {, }}$, R.C. Ball ${ }^{\text {, }}$,

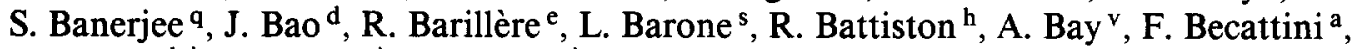
U. Becker ${ }^{\mathrm{k} j}$, F. Behner ${ }^{\mathrm{j}}$, J. Behrens ${ }^{\mathrm{j}}$, S. Beingessner ${ }^{\mathrm{w}}$, Gy.L. Bencze ${ }^{\mathrm{x}}$, J. Berdugo ${ }^{\mathrm{b}}$, P. Berges ${ }^{\mathrm{k}}$,

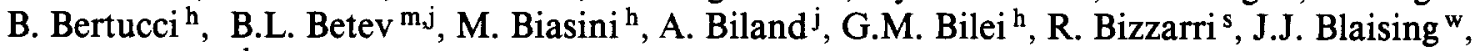
B. Blumenfeld ${ }^{\mathrm{d}}$, G.J. Bobbink ${ }^{\mathrm{e}, \mathrm{y}}$, M. Bocciolini ${ }^{\mathrm{a}}$, R. Bock ${ }^{\mathrm{r}}$, A. Böhm ${ }^{\mathrm{r}}$, B. Borgia ${ }^{\mathrm{s}}$, D. Bourilkov ${ }^{z}$, M. Bourquin ${ }^{\text {v }}$, D. Boutigny ${ }^{\text {w }}$, B. Bouwens ${ }^{y}$, E. Brambilla ${ }^{f}$, J.G. Branson ${ }^{\text {aa }}$, I.C. Brock ${ }^{a b}$, M. Brooks ${ }^{a c}$, C. Buisson ${ }^{\text {ad }}$, A. Bujak ${ }^{\text {ae }}$, J.D. Burger ${ }^{\text {k }}$, W.J. Burger ${ }^{\mathrm{v}}$, J.P. Burq ${ }^{\text {ad }}$, J. Busenitz ${ }^{u}$, X.D. Cai ${ }^{i}$, M. Capell ${ }^{\text {af }}$, M. Caria ${ }^{\text {h }}$, G. Carlino ${ }^{f}$, F. Carminati ${ }^{a}$, A.M. Cartacci ${ }^{a}$, M. Cerrada ${ }^{\text {b }}$, F. Cesaroni ${ }^{\text {, }}$, Y.H. Chang ${ }^{k}$, U.K. Chaturvedi ${ }^{i}$, M. Chemarin ${ }^{\text {ad }}$, A. Chen ${ }^{\text {ag, }}$ C. Chen ${ }^{\text {ah }}$, G.M. Chen ${ }^{\text {ah }}$, H.F. Chen ${ }^{\text {ai }}$, H.S. Chen ${ }^{\text {ah }}$, J. Chen ${ }^{k}$, M. Chen ${ }^{k}$, M.L. Chen ${ }^{p}$, W.Y. Chen ${ }^{i}$, G. Chiefari ${ }^{\text {}}$, C.Y. Chien ${ }^{d}$, M. Chmeissani ${ }^{p}$, S. Chung ${ }^{k}$, C. Civinini ${ }^{a}$, I. Clare ${ }^{k}$,

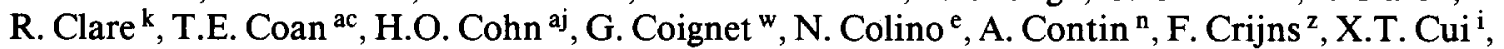
X.Y. Cui ${ }^{i}$, T.S. Dai ${ }^{k}$, R. D’Alessandro ${ }^{a}$, R. de Asmundis ${ }^{f}$, A. Degré ${ }^{w}$, K. Deiters ${ }^{k}$, E. Dénes ${ }^{x}$,

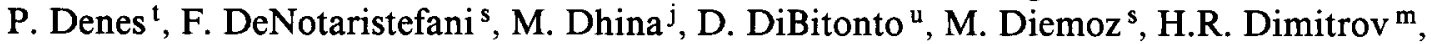
C. Dionisi ${ }^{\text {s,e }}$, M.T. Dova ${ }^{\mathrm{i}}$, E. Drago ${ }^{\mathrm{f}}$, T. Driever ${ }^{\mathrm{z}}$, D. Duchesneau ${ }^{\mathrm{v}}$, P. Duinker ${ }^{\mathrm{y}}$, I. Duran $^{\text {ak }}$, H. El Mamouni ${ }^{\text {ad }}$, A. Engler ${ }^{\text {ab }}$, F.J. Eppling ${ }^{\text {, }}$ F.C. Erné ${ }^{y}$, P. Extermann ${ }^{\mathrm{v}}$, R. Fabbretti ${ }^{a \ell}$, M. Fabre ${ }^{a \ell}$, S. Falciano ${ }^{\text {, }}$, S.J. Fan ${ }^{\text {am }}$, O. Fackler ${ }^{\text {af }}$, J. Fay ${ }^{\text {ad }}$, M. Felcini ${ }^{\text {e }}$, T. Ferguson ${ }^{\text {ab }}$, D. Fernandez ${ }^{\mathrm{b}}$, G. Fernandez ${ }^{\mathrm{b}}$, F. Ferroni $^{\mathrm{s}}$, H. Fesefeldt ${ }^{\mathrm{r}}$, E. Fiandrini ${ }^{\text {h }}{ }^{\text {, J. Field }}{ }^{\mathrm{v}}$, F. Filthaut ${ }^{z}$, G. Finocchiaro ${ }^{\mathrm{s}}$, P.H. Fisher ${ }^{\mathrm{d}}$, G. Forconi ${ }^{\mathrm{v}}$, T. Foreman ${ }^{\mathrm{y}}$, K. Freudenreich ${ }^{\mathrm{j}}$, W. Friebel ${ }^{\text {an }}$, M. Fukushima ${ }^{\text {k }}$, M. Gailloud ${ }^{\text {ao }}$, Yu. Galaktionov ${ }^{\mathrm{o}, \mathrm{k}}$, E. Gallo ${ }^{\mathrm{a}}$, S.N. Ganguli ${ }^{\mathrm{q}}$, P. Garcia-Abia ${ }^{\text {b }, ~ S . S . ~ G a u ~}{ }^{\text {ag }}$, D. Gele ${ }^{\text {ad }}$, S. Gentile ${ }^{\text {s,e }}$, S. Goldfarb ${ }^{\text {g }}$, Z.F. Gong ai, E. Gonzalez ${ }^{\text {b }}$,

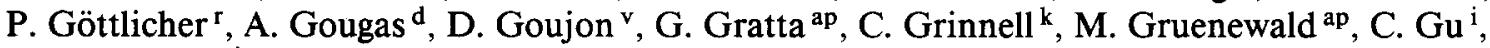
M. Guanziroli ${ }^{i}$, J.K. Guo ${ }^{\text {am }}$, V.K. Gupta ${ }^{t}$, A. Gurtu ${ }^{\text {e,q }}$, H.R. Gustafson ${ }^{\text {p }}$, L.J. Gutay ${ }^{\text {ae }}$, K. Hangarter ${ }^{r}$, A. Hasan ${ }^{i}$, D. Hauschildt ${ }^{y}$, C.F. He ${ }^{\text {am }}$, T. Hebbeker ${ }^{r}$, M. Hebert ${ }^{\text {aa }}$, G. Herten ${ }^{k}$, U. Herten $^{r}$, A. Hervée ${ }^{e}$ K. Hilgers ${ }^{r}$, H. Hofer ${ }^{j}$, H. Hoorani ${ }^{v}$, G. Hu ${ }^{i}$, G.Q. Hu ${ }^{\text {am }}$, B. Ille ${ }^{\text {ad }}$, M.M. Ilyas ${ }^{i}$, V. Innocente ${ }^{e, f}$, H. Janssen ${ }^{e}$, S. Jezequel ${ }^{w}$, B.N. Jin ${ }^{\text {ah }}$, L.W. Jones ${ }^{p}$, A. Kasser ${ }^{\text {ao }}$, R.A. Khan ${ }^{i}$, Yu. Kamyshkov ${ }^{\text {aj }}$, P. Kapinos ${ }^{\ell, a n}$, J.S. Kapustinsky ${ }^{\text {ac }}$, Y. Karyotakis ${ }^{\text {e,w }}$, M. Kaur ${ }^{\mathrm{i}}$, S. Khokhar ${ }^{\text {i }}$, M.N. Kienzle-Focacci ${ }^{\text {v }}$,W.W. Kinnison ${ }^{\text {ac }}$, D. Kirkby ${ }^{\text {ap }, ~ S . ~ K i r s c h ~}{ }^{\text {an }}$, W. Kittel ${ }^{\text {, }}$, A. Klimentov ${ }^{\text {k,o }}$, A.C. König ${ }^{z}$, E. Koffeman ${ }^{\mathrm{y}}$, O. Kornadt ${ }^{\mathrm{r}}$, V. Koutsenko ${ }^{\text {k,o }}$, A. Koulbardis ${ }^{\ell}$, R.W. Kraemer ${ }^{\text {ab }}$, T. Kramer ${ }^{\mathrm{k}}$, V.R. Krastev ${ }^{\mathrm{m}, \mathrm{h}}$, W. Krenz ${ }^{\mathrm{r}}$, A. Krivshich ${ }^{\ell}$, H. Kuijten ${ }^{\mathrm{z}}$, K.S. Kumar ${ }^{a q}$, A. Kunin ${ }^{a q, o}$, G. Landi $^{a}$, D. Lanske ${ }^{r}$, S. Lanzano ${ }^{f}$, P. Lebrun ${ }^{\text {ad }}$, P. Lecomte ${ }^{j}$, P. Lecoq ${ }^{\mathrm{e}}$, P. Le Coultre ${ }^{\mathrm{j}}$, D.M. Lee ${ }^{\text {ac }}$, I. Leedom ${ }^{\mathrm{g}}$, J.M. Le Goffe ${ }^{\mathrm{e}}$, R. Leiste ${ }^{\text {an }}$, M. Lenti ${ }^{\mathrm{a}}$, E. Leonardi ${ }^{\text {s }}$ J. Lettry ${ }^{j}$, X. Leytens ${ }^{y}$, C. Li ${ }^{\text {ai,i }}$, H.T. Li ${ }^{\text {ah }}$, P.J. Li ${ }^{\text {am }}$, X.G. Li ${ }^{\text {ah }}$, J.Y. Liao ${ }^{\text {am }}$, W.T. Lin ${ }^{\text {ag }}$, Z.Y. Lin ${ }^{\text {ai }}$, F.L. Linde ${ }^{\mathrm{e}, \mathrm{y}}$, B. Lindemann ${ }^{\mathrm{r}}$, D. Linnhofer ${ }^{\mathrm{j}}$, L. Lista ${ }^{\mathrm{f}}$, Y. Liu ${ }^{\mathrm{i}}$, W. Lohmann ${ }^{\text {an,e }}$, E. Longo ${ }^{\text {s, Y.S. Lu }}{ }^{\text {ah }}$, J.M. Lubbers ${ }^{\mathrm{e}}$, K. Lübelsmeyer ${ }^{\mathrm{r}}$, C. Luci ${ }^{\text {s }}$, D. Luckey ${ }^{\mathrm{n}, \mathrm{k}}$, 
L. Ludovici $^{\text {s, L. Luminari }}{ }^{\text {s }}$, W. Lustermann ${ }^{\text {an }}$, J.M. Ma ${ }^{\text {ah }}$, W.G. Ma ${ }^{\text {ai }}$, M. MacDermott ${ }^{j}$,

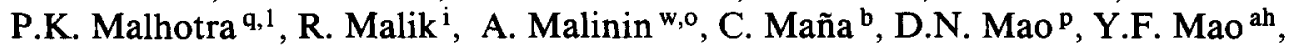

M. Maolinbay ${ }^{j}$, P. Marchesini ${ }^{j}$, F. Marion ${ }^{\text {w }}$, A. Marin ${ }^{\text {c }}$, J.P. Martin ${ }^{\text {ad }}$, L. Martinez-Laso ${ }^{b}$,

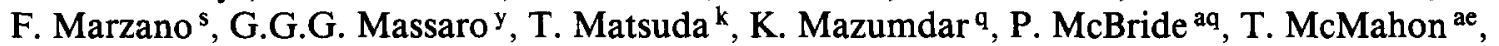
D. McNally ${ }^{j}$, Th. Meinholz ${ }^{r}$, M. Merk ${ }^{z}$, L. Merola ${ }^{f}$, M. Meschini ${ }^{a}$, W.J. Metzger ${ }^{z}$, Y. Mi $^{i}$, G.B. Mills ${ }^{\text {ac }}$, Y. Mir ${ }^{i}$, G. Mirabelli ${ }^{\text {s }}$, J. Mnich ${ }^{r}$, M. Möller ${ }^{r}$, B. Monteleoni ${ }^{a}$, R. Morand ${ }^{\text {w }}$, S. Morganti ${ }^{\text {s, N.E. Moulai }}{ }^{i}$, R. Mount ${ }^{\text {ap }}$, S. Müller ${ }^{r}$, A. Nadtochy ${ }^{\ell}$, E. Nagy ${ }^{x}$, M. Napolitano ${ }^{\text {f }}$, H. Newman ${ }^{\text {ap }}$, C. Neyer ${ }^{j}$, M.A. Niaz ${ }^{i}$, A. Nippe ${ }^{r}$, H. Nowak ${ }^{\text {an }}$, G. Organtini ${ }^{\text {s }}$, D. Pandoulas ${ }^{r}$,

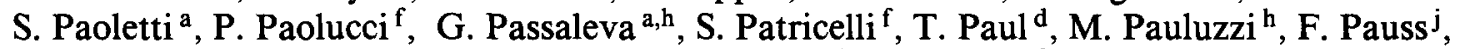
Y.J. Pei ${ }^{r}$, D. Perret-Gallix ${ }^{w}$, J. Perrier ${ }^{v}$, A. Pevsner ${ }^{d}$, D. Piccolo ${ }^{f}$, M. Pieri ${ }^{e, a}$, P.A. Pirouét

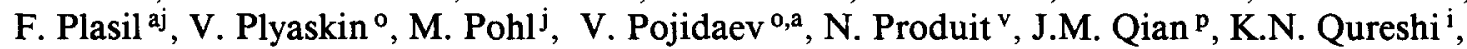

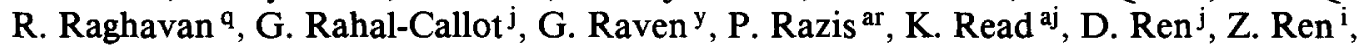
M. Rescigno ${ }^{\text {s }}$, S. Reucroft ${ }^{\mathrm{g}}$, A. Ricker ${ }^{\mathrm{r}}$, S. Riemann ${ }^{\text {an }}$, O. Rind ${ }^{\mathrm{p}}$, H.A. Rizvi ${ }^{\mathrm{i}}$, F.J. Rodriguez ${ }^{\mathrm{b}}$, B.P. Roe ${ }^{\mathrm{p}}$, M. Röhner ${ }^{\mathrm{r}}$, S. Röhner ${ }^{\mathrm{r}}$, L. Romero ${ }^{\mathrm{b}}$, J. Rose ${ }^{\mathrm{r}}$, S. Rosier-Lees ${ }^{\text {w }}$, R. Rosmalen ${ }^{\mathrm{z}}$, Ph. Rosselet ${ }^{\text {ao }}$, A. Rubbia ${ }^{k}$, J.A. Rubio ${ }^{\text {e }}$, H. Rykaczewski ${ }^{j}$, M. Sachwitz ${ }^{\text {an }}$, E. Sajan ${ }^{\text {h }}$, J. Salicio $^{\text {e }}$, J.M. Salicio ${ }^{\text {b }}$, G.S. Sanders ${ }^{\text {ac }}$, A. Santocchia ${ }^{\text {h }}$, M.S. Sarakinos ${ }^{\text {k }}$, G. Sartorelli ${ }^{\text {n,i }}$, M. Sassowsky ${ }^{r}$, G. Sauvage ${ }^{w}$, V. Schegelsky ${ }^{\ell}$, K. Schmiemann ${ }^{r}$, D. Schmitz ${ }^{r}$, P. Schmitz ${ }^{r}$, M. Schneegans ${ }^{\text {w }}$, H. Schopper ${ }^{\text {as }}$, D.J. Schotanus ${ }^{z}$, S. Shotkin ${ }^{k}$, H.J. Schreiber ${ }^{\text {an }}$, J. Shukla $^{\text {ab }}$, R. Schulte ${ }^{r}$, S. Schulte ${ }^{r}$, K. Schultze ${ }^{r}$, J. Schütte ${ }^{a q}$, J. Schwenke ${ }^{r}$, G. Schwering ${ }^{r}$, C. Sciacca ${ }^{f}$, I. Scott $^{\text {aq }}$, R. Sehgal ${ }^{\text {i }}$, P.G. Seiler ${ }^{a \ell}$, J.C. Sens ${ }^{\text {e,y }}$, L. Servoli ${ }^{\text {h }}$, I. Sheer $^{\text {aa }}$, D.Z. Shen ${ }^{\text {am }}$, S. Shevchenko ap, X.R. Shi ${ }^{\text {ap }}$, E. Shumilov ${ }^{\circ}$, V. Shoutko ${ }^{\circ}$, E. Soderstrom ${ }^{t}$, A. Sopczak ${ }^{\text {aa }}$, C. Spartiotis ${ }^{\mathrm{d}}$, T. Spickermann ${ }^{\mathrm{r}}$, P. Spillantini ${ }^{\mathrm{a}}$, R. Starosta ${ }^{\mathrm{r}}$, M. Steuer ${ }^{\mathrm{n}, \mathrm{k}}$, D.P. Stickland ${ }^{\mathrm{t}}$, F. Sticozzi ${ }^{k}$, H. Stone ${ }^{\text {v }}$, K. Strauch ${ }^{\text {aq }}$, B.C. Stringfellow ${ }^{\text {ae }}$, K. Sudhakar ${ }^{\text {q,r }}$, G. Sultanov ${ }^{i}$, R.L. Sumner ${ }^{t}$, L.Z. Sun ${ }^{\text {ai,i }}$, H. Suter ${ }^{j}$, R.B. Sutton ${ }^{\text {ab }}$, J.D. Swain ${ }^{i}$, A.A. Syed ${ }^{i}$, X.W. Tang ${ }^{\text {ah }}$, L. Taylor ${ }^{\mathrm{g}}$, C. Timmermans ${ }^{\mathrm{z}}$, Samuel C.C. Ting ${ }^{\mathrm{k}}$, S.M. Ting ${ }^{\mathrm{k}}$, M. Tonutti ${ }^{\mathrm{r}}$, S.C. Tonwar ${ }^{\mathrm{q}}$, J. Tóth ${ }^{x}$, A. Tsaregorodtsev ${ }^{\ell}$, G. Tsipolitis ${ }^{a b}$, C. Tully ${ }^{\text {ap }}$, K.L. Tung ${ }^{\text {ah }}$, J. Ulbricht ${ }^{j}$, L. Urbán $^{x}$, U. Uwer ${ }^{r}$, E. Valente ${ }^{\mathrm{s}}$, R.T. Van de Walle ${ }^{\mathrm{z}}$, I. Vetlitsky ${ }^{\circ}$, G. Viertel $^{\mathrm{j}}$, P. Vikas ${ }^{\mathrm{i}}$, U. Vikas ${ }^{\mathrm{i}}$, M. Vivargent ${ }^{\text {w }}$, H. Vogel ${ }^{\text {ab }}$, H. Vogt $^{\text {an }}$, I. Vorobiev ${ }^{\circ}$, A.A. Vorobyov ${ }^{\ell}$, L. Vuilleumier $^{\text {ao }}$, M. Wadhwa ${ }^{\mathrm{i}}$, W. Wallraff ${ }^{\mathrm{r}}$, C.R. Wang ${ }^{\text {ai }}$, G.H. Wang ${ }^{\text {ab }}$, J.H. Wang ${ }^{\text {ah }}$, Q.F. Wang ${ }^{\text {aq }}$, X.L. Wang ${ }^{\text {ai }}$, Y.F. Wang ${ }^{\text {a }}$, Z.M. Wang ${ }^{\text {i,ai }}$, A. Weber ${ }^{r}$, J. Weber ${ }^{j}$, R. Weill ${ }^{\text {ao }}$, T.J. Wenaus ${ }^{\text {af }}$, J. Wenninger $^{\mathrm{v}}$, M. White ${ }^{\mathrm{k}}$, C. Willmott ${ }^{\mathrm{b}}$, F. Wittgenstein ${ }^{\mathrm{e}}$, D. Wright ${ }^{\mathrm{t}}$, R.J. Wu ${ }^{\text {ah }}$, S.X. Wu ${ }^{\mathrm{i}}$, Y.G. Wu ah , B. Wyslouch ${ }^{k}, Y . Y . X e^{a m}, Y . D . X u^{\text {ah }}, Z . Z$. Xu ${ }^{\text {ai }}$, Z.L. Xue ${ }^{\text {am }}$, D.S. Yan ${ }^{\text {am }}$, X.J. Yan ${ }^{\text {k, B.Z. Yang }}{ }^{\text {ai }}$, C.G. Yang ${ }^{\text {ah }}$, G. Yang ${ }^{\mathrm{i}}$, K.S. Yang ${ }^{\text {ah }}$, Q.Y. Yang ${ }^{\text {ah }}$, Z.Q. Yang ${ }^{\text {am }}$, C.H. Ye ${ }^{\mathrm{i}}$, J.B. Ye ${ }^{\text {ai }}$, Q. Ye ${ }^{\mathrm{i}}$, S.C. Yeh ${ }^{\text {ag }}$, Z.W. Yin ${ }^{\text {am }}$, J.M. You $^{\mathrm{i}}$, N. Yunus ${ }^{\mathrm{i}}$, M. Yzerman ${ }^{\mathrm{y}}$, C. Zaccardelli ap , P. Zemp ${ }^{j}$, M. Zeng ${ }^{\mathrm{i}}$, Y. Zeng ${ }^{\mathrm{r}}$, D.H. Zhang ${ }^{\mathrm{y}}$, Z.P. Zhang ${ }^{\text {ai,i }}$, B. Zhou ${ }^{\mathrm{c}}$, J.F. Zhou ${ }^{\text {r }}$, R.Y. Zhu ${ }^{\text {ap }}$, H.L. Zhuang ${ }^{\text {ah }}$, A. Zichichi ${ }^{\text {ne, }}{ }^{\text {a }}$ and B.C.C. van der Zwaan ${ }^{y}$

a INFN - Sezione di Firenze and Università di Firenze, I-50125 Florence, Italy

b Centro de Investigaciones Energeticas, Medioambientales y Tecnologicas, CIEMAT, E-28040 Madrid, Spain

c Boston University, Boston, MA 02215, USA

d Johns Hopkins University, Baltimore, MD 21218, USA

e European Laboratory for Particle Physics, CERN, CH-1211 Geneva 23, Switzerland

f INFN - Sezione di Napoli and Università di Napoli, I-80125 Naples, Italy

Northeastern University, Boston, MA 02115, USA

h INFN - Sezione di Perugia and Università Degli Studi di Perugia, I-06100 Perugia, Italy

i World Laboratory, FBLJA Project, CH-1211 Geneva 23, Switzerland

j Eidgenössische Technische Hochschule, ETH Zürich, CH-8093 Zurich, Switzerland

k Massachusetts Institute of Technology, Cambridge, MA 02139, USA

${ }^{\ell}$ Nuclear Physics Institute, St. Petersburg, Russian Federation 
$\mathrm{m}$ Bulgarian Academy of Sciences, Institute of Mechatronics, BU-1113 Sofia, Bulgaria

" INFN - Sezione di Bologna, I-40126 Bologna, Italy

- Institute of Theoretical and Experimental Physics, ITEP, 117259 Moscow, Russian Federation

p University of Michigan, Ann Arbor, MI 48109, USA

q Tata Institute of Fundamental Research, Bombay 400 005, India

I. Physikalisches Institut, RWTH, W-5100 Aachen, FRG ${ }^{2}$ and III. Physikalisches Institut, RWTH, W-5100 Aachen, FRG ${ }^{2}$

s INFN - Sezione di Roma and Università di Roma "La Sapienza", I-00185 Rome, Italy

'Princeton University, Princeton, NJ 08544, USA

"University of Alabama, Tuscaloosa, AL 35486, USA

University of Geneva, CH-1211 Geneva 4, Switzerland

" Laboratoire de Physique des Particules, LAPP, F-74941 Annecy-le-Vieux, France

x Central Research Institute for Physics of the Hungarian Academy of Sciences, H-1525 Budapest 114, Hungary

y National Institute for High Energy Physics, NIKHEF, NL-1009 DB Amsterdam. The Netherlands

${ }^{z}$ University of Nijmegen and NIKHEF, NL-6525 ED Nijmegen, The Netherlands

aa University of California, San Diego, CA 92182, USA

ab Carnegie Mellon University, Pittsburgh, PA 15213, USA

ac Los Alamos National Laboratory, Los Alamos, NM 87544, USA

ad Institut de Physique Nucléaire de Lyon, IN2P3-CNRS/Université Claude Bernard, F-69622 Villeurbanne Cedex, France

ae Purdue University, West Lafayette, IN 47907, USA

af Lawrence Livermore National Laboratory, Livermore, CA 94550, USA

as High Energy Physics Group, Taiwan, ROC

ah Institute of High Energy Physics, IHEP, Beijing, China

ai Chinese University of Science and Technology, USTC, Hefei, Anhui 230 029, China

aj Oak Ridge National Laboratory, Oak Ridge, TN 37830, USA

ak Departamento de Fisica de Particulas Elementales, Universidad de Santiago, E-15706 Santiago de Compostela, Spain

al Paul Scherrer Institut, PSI, CH-5232 Villigen, Switzerland

am Shanghai Institute of Ceramics, SIC, Shanghai, China

an DESY-Institut für Hochenergiephysik, O-1615 Zeuthen, FRG

ao University of Lausanne, CH-1015 Lausanne, Switzerland

ap California Institute of Technology, Pasadena, CA 91125, USA

aq Harvard University, Cambridge, MA 02139, USA

ar Department of Natural Sciences, University of Cyprus, Nicosia, Cyprus

as University of Hamburg, W-2000 Hamburg, FRG

Received 21 July 1992

We have measured the forward-backward asymmetry in $\mathrm{e}^{+} \mathrm{e}^{-} \rightarrow \mathrm{b} \overline{\mathrm{b}}$ and $\mathrm{e}^{+} \mathrm{e}^{-} \rightarrow \mathrm{c} \overline{\mathrm{c}}$ processes using hadronic events containing muons or electrons. The data sample corresponds to 410000 hadronic decays of the $Z^{0}$. From a fit to the single lepton and dilepton $p$ and $p_{\mathrm{T}}$ spectra, we determine $A_{\mathrm{b} \overline{\mathrm{b}}}=0.086 \pm 0.015 \pm 0.007$ and $A_{\mathrm{c} \overline{\mathrm{c}}}=0.083 \pm 0.038 \pm 0.027$ at the effective center-of-mass energy $\sqrt{s}=91.24 \mathrm{GeV}$. These measurements yield a value of the electroweak mixing angle $\sin ^{2} \bar{\theta}_{\mathrm{w}}=0.2336 \pm 0.0029$.

\section{Introduction}

The forward-backward asymmetry of quark pairs, $A_{\mathrm{q} \overline{\mathrm{q}}}$, produced in the $\mathrm{e}^{+} \mathrm{e}^{-} \rightarrow \mathrm{Z}^{0} \rightarrow \mathrm{q} \overline{\mathrm{q}}$ process is sensitive to the electroweak mixing angle, $\sin ^{2} \theta_{\mathrm{w}}$, which

1 Deceased.

2 Supported by the German Bundesministerium für Forschung und Technologie. is one of the fundamental parameters of the standard model [1]. The forward-backward asymmetry for the process $\mathrm{e}^{+} \mathrm{e}^{-} \rightarrow \mathrm{q} \overline{\mathrm{q}}$ is defined as

$A_{\mathrm{q} \tilde{\mathrm{q}}}=\frac{\sigma_{\mathrm{F}}^{\mathrm{q}}-\sigma_{\mathrm{B}}^{\mathrm{q}}}{\sigma_{\mathrm{F}}^{\mathrm{q}}+\sigma_{\mathrm{B}}^{\mathrm{q}}}$,

where $\sigma_{\mathrm{F}}^{\mathrm{q}}$ and $\sigma_{\mathrm{B}}^{\mathfrak{q}}$ are the cross-sections in the forward and backward hemispheres with respect to the electron beam. In the standard model using the improved 
Born approximation framework $[2,3]$ the asymmetry on the $Z^{0}$ peak is given by

$A_{\bar{q} \overline{\mathrm{q}}}^{\text {Born }} \equiv \frac{3}{4} A_{\mathrm{e}} A_{\mathrm{q}}$,

where

$A_{i}=\frac{2 v_{i} a_{i}}{v_{i}^{2}+a_{i}^{2}}=\frac{2\left(1-4\left|Q_{i}\right| \sin ^{2} \bar{\theta}_{w}\right)}{1+\left(1-4\left|Q_{i}\right| \sin ^{2} \bar{\theta}_{w}\right)^{2}}$.

Here $v_{i}, a_{i}$ and $Q_{i}$ are the vector and axial-vector coupling constants and the charge of the electron or quark, and $\sin ^{2} \bar{\theta}_{w}$ is the effective electroweak mixing angle at the $Z^{0}$ mass. The resulting angular distribution of the quark is

$$
\frac{\mathrm{d} \sigma}{\mathrm{d} \cos \theta} \propto \frac{3}{8}\left(1+\cos ^{2} \theta\right)+A_{\mathrm{q} \overline{\mathrm{q}}} \cos \theta,
$$

where $\theta$ is the polar angle of the quark with respect to the electron beam.

In this letter we present measurements of $A_{\mathrm{bb}}$ and $A_{\mathrm{cc}}$. We use electrons and muons from the semileptonic decay of $b$ - or c-quarks to select events coming from $Z^{0} \rightarrow b \bar{b}$ and $Z^{0} \rightarrow c \bar{c}$. Because of the hard fragmentation and large mass of the b-quark, leptons from b-quark decay have large momentum, $p$, and large transverse momentum, $p_{\mathrm{T}}$, with respect to the quark direction. The c-quark, with its lower mass and softer fragmentation, produces leptons with lower $p$ and $p_{\mathrm{T}}$, but nevertheless still higher than that of leptons from the decays of the lighter quarks. As the charge of the lepton is correlated with the charge of the quark, we can use events containing these inclusive leptons to measure $A_{\mathrm{bb}}$ and $A_{\mathrm{c} \overline{\mathrm{c}}}$. We use the thrust axis of the event to give the direction of the quark and we tag its charge with the lepton charge. To determine $A_{\mathrm{bb}}$ and $A_{\mathrm{cc}}$, we perform a maximum likelihood fit to the single lepton and dilepton $p$ and $p_{\mathrm{T}}$ spectra. Similar analyses have previously been performed with lower statistics [4-8].

Due to mixing in the $\mathrm{B}^{0}-\mathrm{B}^{0}$ system, the observed b-quark asymmetry, $A_{\mathrm{bb}}^{\mathrm{obs}}$, is smaller than the actual asymmetry by a factor $\left(1-2 \chi_{\mathrm{B}}\right)$, where $\chi_{\mathrm{B}}$ is the probability that a hadron containing a b-quark had oscillated into a hadron containing a $\bar{b}$-quark at the time of its decay. We have reported a measurement of $\chi_{\mathrm{B}}$ in a previous letter [9]. As there is no observable mixing with $\mathrm{D}$ mesons, $A_{\mathrm{c} \bar{c}}$ is measured directly.

Our data sample consists of 410000 hadronic events corresponding to $17.6 \mathrm{pb}^{-1}$ collected in 1990 and 1991 on or near the $Z^{0}$ resonance using the $\mathrm{L} 3$ detector at LEP. The center-of-mass energies are distributed over the range $88.2 \leqslant \sqrt{s} \leqslant 94.2 \mathrm{GeV}$.

\section{The $\mathbf{L 3}$ detector}

The L3 detector covers $99 \%$ of $4 \pi$. The detector consists of a central tracking chamber, a high resolution electromagnetic calorimeter composed of BGO crystals, a ring of scintillation counters, a uranium and brass hadron calorimeter with proportional wire chamber readout, and an accurate muon chamber system. These detectors are installed in a $12 \mathrm{~m}$ diameter magnet which provides a uniform field of $0.5 \mathrm{~T}$ along the beam direction.

The central tracking chamber is a time expansion chamber which consists of two cylindrical layers of 12 and 24 sectors, with 62 wires measuring the $R-\phi$ coordinate. The single wire resolution is $58 \mu \mathrm{m}$ averaged over the entire cell. The double-track resolution is $640 \mu \mathrm{m}$. The fine segmentation of the BGO detector and the hadron calorimeter allows us to measure the direction of jets with an angular resolution of $2.5^{\circ}$, and to measure the total energy of hadronic events from $Z^{0}$ decay with a resolution of $10 \%$. The muon detector consists of 3 layers of precise drift chambers which measure 56 points on the muon trajectory in the bending plane, and 8 points in the non-bending direction.

For the present analysis, we use the data collected in the following ranges of polar angles:

- for the central chamber, $41^{\circ}<\theta<139^{\circ}$,

- for the hadron calorimeter, $5^{\circ}<\theta<175^{\circ}$,

- for the muon chambers, $35.8^{\circ}<\theta<144.2^{\circ}$,

- for the electromagnetic calorimeter, $11^{\circ}<\theta<$ $169^{\circ}$.

A detailed description of each detector subsystem, and its performance, is given in ref. [10].

\section{Selection of inclusive lepton events}

The trigger requirements and the selection criteria for hadronic events containing electrons and muons have been described earlier $[9,11]$. Muons are identified and measured in the muon chamber system. We require that a muon track consist of track segments in 
at least two of the three layers of muon chambers, and that the muon track point to the intersection region. Electrons are identified using the BGO and hadron calorimeters, as well as the central tracking chamber. We require an energy cluster in the BGO that is consistent with the shape of an electromagnetic shower and which matches in $\phi$ angle and momentum with a track in the central tracking chamber. For this analysis, we have only considered electrons in the barrel region $(|\cos \theta|<0.69)$. We reject hadrons misidentified as electrons by requiring that there be less than $3 \mathrm{GeV}$ deposited in the hadron calorimeter in a cone of half angle $7^{\circ}$ behind the electromagnetic cluster. The charge of the electron is determined from the tracking chamber. The shower shape and hadron calorimeter criteria select electrons that are isolated from nearby particles, resulting in a lower efficiency for electrons than for muons.

The momentum of muon candidates is required to be at least $4 \mathrm{GeV}$, while the electrons are required to have at least $3 \mathrm{GeV}$. Table 1 shows the number of single lepton and dilepton events obtained after all cuts.

To determine the acceptance for inclusive lepton events, we use the JETSET parton shower program [12] with string fragmentation. For b- and c-quarks we use the Peterson fragmentation function [13] as a function of $x_{E}=2 E_{\text {hadron }} / \sqrt{s}$ with the parameters $\epsilon_{\mathrm{b}}=0.05$ and $\epsilon_{\mathrm{c}}=0.50$. The generated events are passed through the L3 detector simulation $^{\# 1}$, which includes the effects of energy loss, multiple scattering, interactions and decays in the detector materials and beam pipe. We use the average of the semileptonic branching ratios measured by

\#1 The L3 detector simulation is based on GEANT Version 3.14 [14]. The GHEISHA program [15] is used to simulate hadronic interactions.

Table 1

Number of inclusive lepton events.

\begin{tabular}{lr}
\hline Type & Events \\
\hline$\mu+$ hadrons & 20937 \\
$\mathrm{e}+$ hadrons & 5443 \\
$\mu \mu+$ hadrons & 1083 \\
ee + hadrons & 152 \\
$\mu \mathrm{e}+$ hadrons & 709 \\
\hline
\end{tabular}
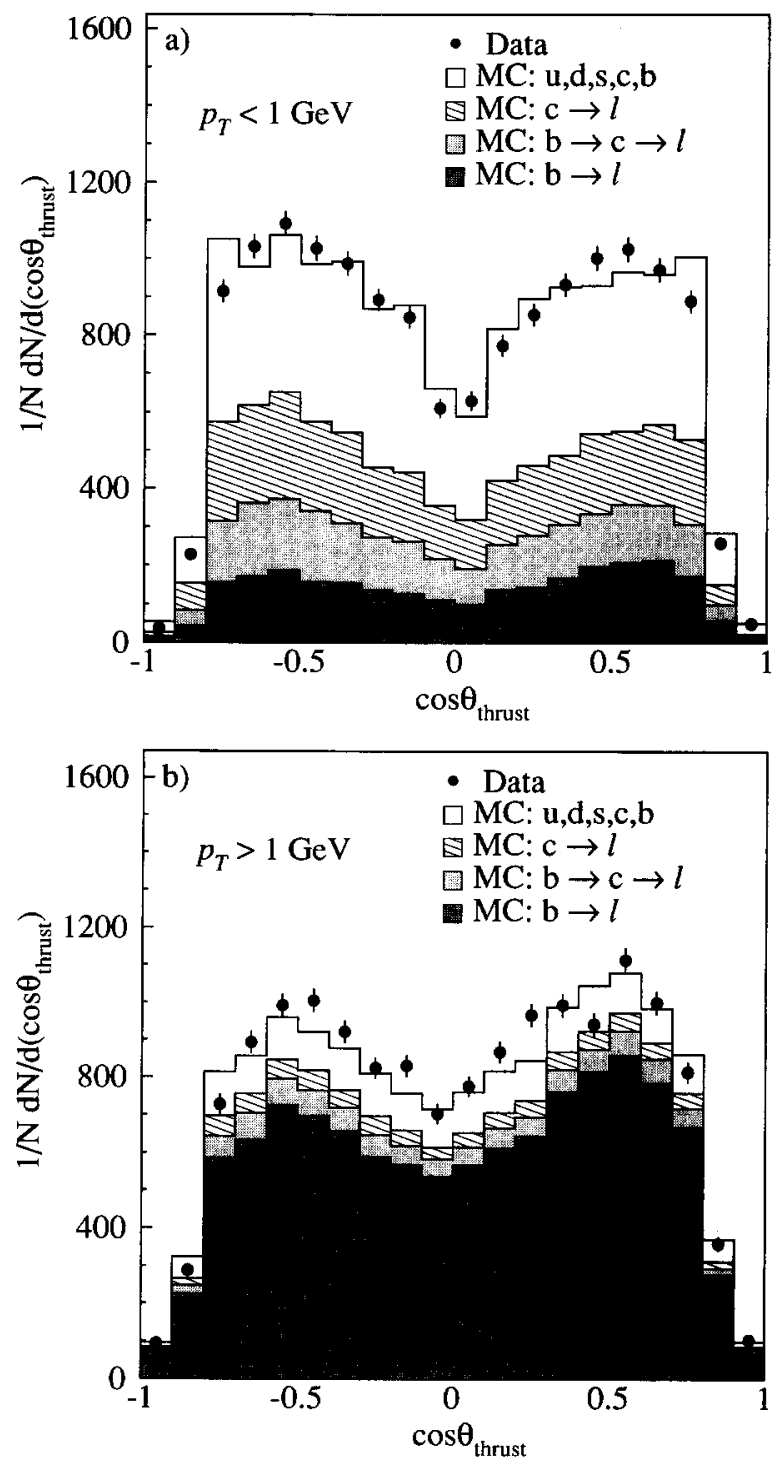

Fig. 1. The measured angular distribution of the thrust axis for events containing a low $p_{\mathrm{T}}(<1 \mathrm{GeV})$ lepton (a) or a high $p_{\mathrm{T}}$ lepton $(>1 \mathrm{GeV}$ ) (b). The contributions of the various sources are indicated.

previous experiments $[16,17]^{\# 2}: \operatorname{Br}(\mathrm{b} \rightarrow \ell)=$

\#2 We have averaged the PETRA and PEP measurements according to the procedure used by the Particle Data Group.

\#3 The notation $b \rightarrow \ell$ means the decay of a hadron containing a b-quark into a lepton (plus its neutrino) and anything else. Similarly for $\mathrm{c} \rightarrow \ell$, etc. Charge-conjugate processes are also implied. 
Table 2

Monte Carlo estimates of the fraction of each process in the data sample (in percent). Also shown is the expected asymmetry for each process.

\begin{tabular}{llllll}
\hline Category & $\mu$ & & $\mathrm{e}$ & \\
\cline { 2 - 3 } \cline { 5 - 6 } & $p_{\mathrm{T}}>0 \mathrm{GeV}$ & $p_{\mathrm{T}}>1 \mathrm{GeV}$ & & $p_{\mathrm{T}}>0 \mathrm{GeV}$ & $p_{\mathrm{T}}>1 \mathrm{GeV}$ \\
\hline$(1) \mathrm{b} \rightarrow \ell$ & 36.5 & 70.5 & 67.6 & 79.8 \\
$(2) \mathrm{b} \rightarrow \mathrm{c} \rightarrow \ell$ & 10.8 & 6.6 & 6.6 & 4.3 \\
$(3) \mathrm{b} \rightarrow \tau \rightarrow \ell$ & 1.8 & 1.8 & 2.5 & 2.0 \\
$(4) \mathrm{b} \rightarrow \overline{\mathrm{c}} \rightarrow \ell$ & 1.3 & 0.6 & 0.7 & 0.4 \\
$(5) \mathrm{c} \rightarrow \ell$ & 16.1 & 14.0 & & 4.3 & 2.5 \\
(6) background & 33.5 & & 18.3 & 11.1 \\
\hline
\end{tabular}

$0.117 \pm 0.006$ and $\operatorname{Br}(\mathrm{c} \rightarrow \ell)=0.096 \pm 0.006$. We assume that the mixture of $\mathrm{c}$-hadrons from the cascade decay $b \rightarrow c$ is the same as that in $Z^{0} \rightarrow c \bar{c}$, and so we use the measured $\mathrm{c} \rightarrow \ell$ branching ratio also for $\mathrm{b} \rightarrow \mathrm{c} \rightarrow \ell$, after taking into account the additional c-quarks coming from $W$ decays. We determine that the efficiency for observing a prompt $b \rightarrow \ell$ decay is $42.5 \%$ for muons and $23.3 \%$ for electrons.

Monte Carlo events with leptons are classified into six categories: $\mathrm{b} \rightarrow \ell, \mathrm{b} \rightarrow \mathrm{c} \rightarrow \ell, \mathrm{b} \rightarrow \tau \rightarrow \ell, \mathrm{b} \rightarrow$ cc̄s where $\overline{\mathrm{c}} \rightarrow \ell, \mathrm{c} \rightarrow \ell$, and background. Included in the background are leptons from $\pi$ and $\mathrm{K}$ decays, Dalitz decays, photon conversions, and misidentified hadrons caused by, for example, $\pi-\gamma$ overlap for electrons and punchthrough for muons. Table 2 shows the results of Monte Carlo studies giving the fraction of each source of leptons and background for data samples with no cut on transverse momentum and also with a cut at $1.0 \mathrm{GeV}$. The transverse momentum is defined with respect to the nearest jet [18], where the measured energy of the lepton is excluded from the jet. If there is no jet with an energy greater than 6 $\mathrm{GeV}$ remaining in the same hemisphere as the lepton, then the $p_{\mathrm{T}}$ is calculated relative to the thrust axis of the event.

As can be seen from table 2, the data at high $p_{\mathrm{T}}$ for both electrons and muons are dominated by events containing b-quarks. At low $p_{\mathrm{T}}$ the muon sample has a relatively large contribution from c-quarks. This is also illustrated in fig. 1 which shows the angular distribution of the thrust axis for low and high $p_{\mathrm{T}}$ events. The requirements of the electron selection result in a reduced efficiency for low $p_{\mathrm{T}}$ events, and hence for c-quark events.

\section{Determination of the forward-backward asymmetries}

In the semileptonic decay of a b- or c-quark the charge of the detected lepton is directly correlated with the charge of the quark. We use the thrust axis to estimate the direction of the original quark. The thrust axis is oriented towards the hemisphere containing the negatively charged lepton (or opposite the positively charged lepton ). With this convention, the thrust axis points in the direction of the b-quark for $b \bar{b}$ events and in the direction of the $\bar{c}$-quark for cic events.

We first investigate the angular distribution of the thrust axis for high $p_{\mathrm{T}}(>1 \mathrm{GeV})$ events. In this way, we can make a direct measurement of $A_{\mathrm{bb}}^{\text {obs }}$. The data are corrected for angular acceptance, and the non-bquark background is subtracted. The resulting distribution is shown in fig. 2. A fit is made to the form:

$\frac{3}{8}\left(1+\cos ^{2} \theta_{\text {thrust }}\right)+A_{\mathrm{bb}}^{\text {obs }} \cos \theta_{\text {thrust }}$.

The result is $A_{\mathrm{bb}}^{\mathrm{obs}}=0.062 \pm 0.013$ with $\chi^{2} / \mathrm{DOF}=$ $26 / 19$. This method, however, has the disadvantage that the $p$ and $p_{\mathrm{T}}$ of the lepton are not used, thus reducing sensitivity. In addition, this method is best suited to situations where the background is small, and is therefore unsuitable for a measurement of $A_{\mathrm{c} c}$.

To use the full statistics and improve the sensitivity, we determine $A_{\mathrm{bb}}$ and $A_{\mathrm{c} \bar{c}}$ using a non-binned maximum likelihood fit in the $p$ versus $p_{\mathrm{T}}$ plane. The fitting procedure has been described in detail previously [5]. The probability for a data event to come from the various sources listed in table 2 is determined from the number and type of Monte Carlo events found in a rectangular box centered on the $\left(p, p_{\Upsilon}\right)$ values of the data event. The weights for the likelihood function 


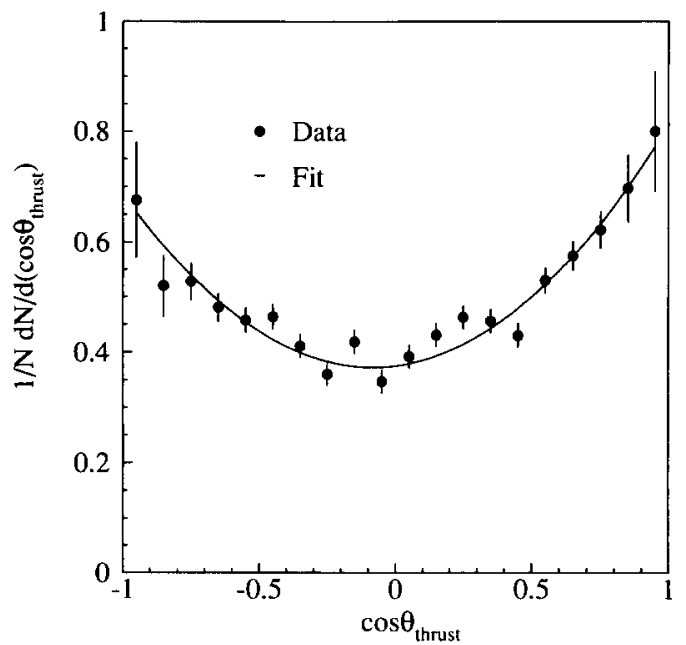

Fig. 2. The angular distribution of the thrust axis for events containing high $p_{\mathrm{T}}$ leptons. The orientation of the thrust axis has been described in the text. The points are the data, and the solid line is the result of the fit.

are determined by the expected angular distribution given the polar angle of the thrust axis of the data event, and the asymmetry of the possible sources of the event. For sources 1,3 and 4, the asymmetry is $A_{\mathrm{bb}}$, while for source 2 it is $-A_{\mathrm{bb}}$, as the lepton from the cascade has the opposite sign than the b-quark. For source 5 , it is $-A_{\mathrm{c} \bar{c}}$, as the thrust axis points in the direction of the $\bar{c}$-quark. In the case of dilepton events, the average asymmetries of the two leptons are used.

From a sample of $\mathrm{Z}^{0} \rightarrow \tau^{+} \tau^{-}$events we estimate the charge confusion to be $0.2 \pm 0.2 \%$ for muons and $0.8 \pm 0.3 \%$ for electrons. We correct for the effects of the charge confusion, and account for its error in the systematic error.

The result of the fit for $A_{\mathrm{b} \bar{b}}$ and $A_{\mathrm{c} \bar{c}}$ using both inclusive muons and electrons and all center-of-mass energies is $A_{\mathrm{bb}}^{\mathrm{obs}}=0.066 \pm 0.011$ and $A_{\mathrm{c} \overline{\mathrm{c}}}=0.083 \pm 0.038$. As the acceptance for $\mathrm{c} \rightarrow \mathrm{e}$ events is small, the $A_{\mathrm{cc}}$ measurement is determined almost completely by the inclusive muon events. The correlation coefficient between $A_{\mathrm{bb}}^{\mathrm{obs}}$ and $A_{\mathrm{cc}}$ is $20 \%$. Separate fits for $A_{\mathrm{bb}}$ using the muon and electron data yield $A_{\mathrm{bb}}^{\mathrm{obs}}=0.074 \pm 0.014$ for muons, and $0.053 \pm 0.019$ for electrons.

Table 3 lists the contributions to the systematic error in the $A_{\mathrm{b} \bar{b}}^{\mathrm{obs}}$ and $A_{\mathfrak{c} \bar{c}}$ measurements. We have estimated the error by changing the parameters by their known (or estimated) uncertainties. The $c \rightarrow \ell$

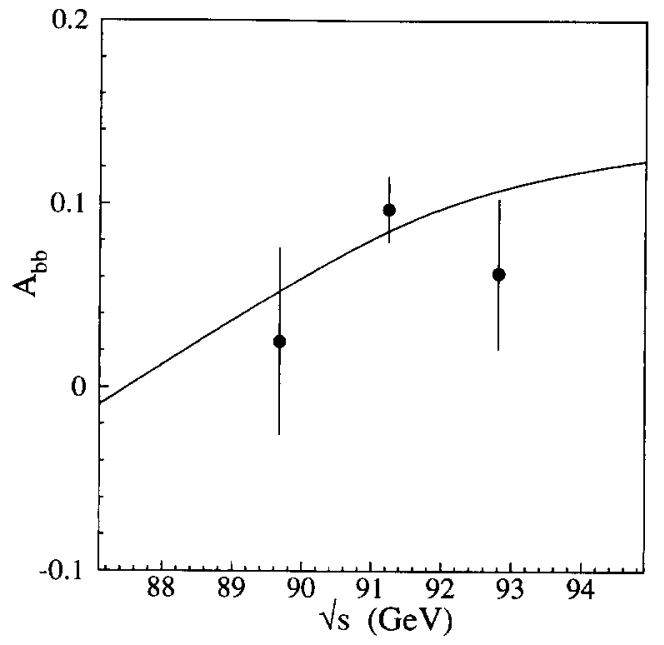

Fig. 3. The energy dependence of $A_{\mathrm{b} b}$ compared to the standard model expectation with $\sin ^{2} \bar{\theta}_{\mathrm{w}}=0.2336$.

branching ratio error has been increased to \pm 0.012 to allow for uncertainties in the $b \rightarrow c \bar{c} s$ branching ratio, as well as a possible different mixture of $D^{ \pm}$ and $D^{0}$ mesons in b-quark decays. The charge of the background can be correlated with the charge of the original quark, and therefore background processes could affect the measured asymmetry. We estimate this correlation to be $0.35 \pm 0.15$ for background from b-quarks, and negligible for the lighter quarks. The background asymmetry for non-b-quark sources was determined from Monte Carlo studies to be consistent with zero. The error on this estimate is 0.015 which gives the dominant systematic error for $A_{\mathrm{cc}}$. We have estimated the effect of reconstruction errors by smearing the momentum of each lepton by $5 \%$. The effects of jet angular resolution have been studied by smearing the jet directions in the Monte Carlo with a Gaussian distribution with a width between $0^{\circ}$ and $1^{\circ}$.

Our result for $A_{\bar{c} \mathfrak{c}}$ is

$A_{\mathrm{cc}}=0.083 \pm 0.038$ (stat.) \pm 0.027 (syst.).

Due to mixing in the $\mathrm{B}^{0}-\mathrm{B}^{0}$ system, the observed $\mathrm{b} \overrightarrow{\mathrm{b}}$ asymmetry is $A_{\mathrm{bb}}^{\mathrm{obs}}=A_{\mathrm{bb}}\left(1-2 \chi_{\mathrm{B}}\right)$. Correcting $A_{\mathrm{bb}}^{\mathrm{obs}}$ using our measured value $\chi_{\mathrm{B}}=0.121 \pm 0.017$ (stat.) \pm 0.006 (syst.) [9] we obtain

$A_{\mathrm{bb}}=0.086 \pm 0.015$ (stat.) \pm 0.007 (syst.). 
Table 3

Systematic errors in the $A_{\mathrm{b} \overline{\mathrm{b}}}^{\mathrm{obs}}$ and $A_{\mathrm{c} \overline{\mathrm{c}}}$ measurements.

\begin{tabular}{lccc}
\hline Contribution & Variation & $\Delta A_{\mathrm{bb}}^{\text {obs }}$ & $\Delta A_{\mathrm{cc}}$ \\
\hline $\mathrm{b} \rightarrow \ell$ branching ratio & \pm 0.006 & 0.0006 & 0.002 \\
$\mathrm{c} \rightarrow \ell$ branching ratio & \pm 0.012 & 0.0013 & 0.013 \\
$\Gamma_{\mathrm{b} \overline{\mathrm{b}}}$ & $\pm 10 \mathrm{MeV}$ & 0.0003 & 0.001 \\
$\Gamma_{\mathrm{c} \overline{\mathrm{c}}}$ & $\pm 10 \mathrm{MeV}$ & 0.0002 & 0.002 \\
background fraction & \pm 0.15 & 0.0006 & 0.003 \\
background asymmetry & \pm 0.015 & 0.0006 & 0.020 \\
charge correlation for b-quark background & \pm 0.15 & 0.0004 & 0.001 \\
b-quark fragmentation parameter $\epsilon_{\mathrm{b}}$ & \pm 0.006 & 0.0002 & 0.002 \\
c-quark fragmentation parameter $\epsilon_{\mathrm{c}}$ & \pm 0.200 & 0.0006 & 0.004 \\
smearing the lepton momentum $\Delta p / p$ & $5 \%$ & 0.0001 & 0.001 \\
smearing the angle between the lepton and nearest jet & $00-1{ }^{\circ}$ & 0.0010 & 0.004 \\
uncertainty in the charge confusion correction & \pm 0.0015 & 0.0002 & 0.001 \\
Monte Carlo statistics & & 0.0035 & 0.009 \\
total & & 0.004 & 0.027 \\
\hline
\end{tabular}

Table 4

$A_{\mathrm{bb}}$ for different center-of-mass energies. Note that the systematic error 0.007 is common to all energies.

\begin{tabular}{ll}
\hline Mean $\sqrt{s}(\mathrm{GeV})$ & $A_{\mathrm{bb}}$ \\
\hline 89.67 & $0.025 \pm 0.051 \pm 0.007$ \\
91.24 & $0.097 \pm 0.017 \pm 0.007$ \\
92.81 & $0.062 \pm 0.042 \pm 0.007$ \\
\hline
\end{tabular}

As the asymmetry is predicted to depend on the center-of-mass energy, we also have divided our data into energies below, on, and above the $Z^{0}$ resonance. The results for $A_{\mathrm{bb}}$ are shown in table 4 and fig. 3 . Because of limited statistics, we have not performed this measurement for $A_{\mathrm{cc}}$.

\section{Determination of $\sin ^{2} \bar{\theta}_{w}$}

The Born level approximation given in the introduction is only valid at the $\mathrm{Z}^{0}$ mass. In addition, one must apply QED initial and final state radiative corrections, as well as QCD corrections due to gluon bremsstrahlung. We use the ZFITTER program [19] to perform these corrections and extract $\sin ^{2} \bar{\theta}_{w}$. As input we use our measured values [20] of $m_{\mathrm{Z}}=$ $91.181 \pm 0.022 \mathrm{GeV}$ and $\Gamma_{\mathrm{Z}}=2.501 \pm 0.017 \mathrm{GeV}$, and the asymmetry values and energies given in table 4 . We obtain $\sin ^{2} \bar{\theta}_{w}=0.2336 \pm 0.0029$,

which is in excellent agreement with our measurement [20] of $\sin ^{2} \bar{\theta}_{\mathrm{w}}=0.2315 \pm 0.0025$ from the leptonic and hadronic decays of the $Z^{0}$. Fig. 3 shows the measured energy dependence of $A_{\mathrm{b} b}$ in comparison with the standard model expectation with $\sin ^{2} \bar{\theta}_{w}=$ 0.2336 . The standard model prediction for $A_{\mathrm{cc}}$ is 0.056 , in good agreement with our measurement.

As can be seen in fig. 3 , the asymmetry predicted by the standard model is not linear as a function of energy, but rather flattens out above the $Z^{0}$ peak due to initial state radiation. We have used ZFITTER to take this effect into account, and to calculate the effective average center-of-mass energy for our complete data sample also taking into account the luminosity and cross-section at each energy. As most of our data was taken on the $Z^{0}$ peak, and as the effect is small, the effective average is the same as the peak energy: 91.24 $\mathrm{GeV}$.

\section{Conclusions}

We have analyzed $Z^{0} \rightarrow b \bar{b}$ and $Z^{0} \rightarrow c \bar{c}$ decays using inclusive lepton events selected from a sample of 410000 hadronic events.

From a fit to the $p$ and $p_{\mathrm{T}}$ distributions for single lepton and dilepton events, we have determined the $b \bar{b}$ 
and cć forward-backward asymmetries at the effective center-of-mass energy $\sqrt{s}=91.24 \mathrm{GeV}$ to be

$A_{\mathrm{b} \overline{\mathrm{b}}}=0.086 \pm 0.015 \pm 0.007$,

$A_{\mathrm{c} \overline{\mathrm{c}}}=0.083 \pm 0.038 \pm 0.027$

Using the values of $A_{\mathrm{bb}}$ obtained below, on, and above the $Z^{0}$ peak, we have determined $\sin ^{2} \bar{\theta}_{w}$ to be $\sin ^{2} \bar{\theta}_{\mathrm{w}}=0.2336 \pm 0.0029$.

\section{Acknowledgement}

We wish to express our gratitude to the CERN accelerator divisions for the excellent performance of the LEP machine. We acknowledge the contributions of all the engineers and technicians who have participated in the construction and maintenance of this experiment. Those of us who are not from member states thank CERN for its hospitality and help.

\section{References}

[1] S.L. Glashow, Nucl. Phys. 22 (1961) 579; S. Weinberg, Phys. Rev. Lett. 19 (1967) 1264; A. Salam, Elementary particle theory, ed. N. Svartholm (Almquist and Wiksell, Stockholm, 1968) p. 367.

[2] J. Kühn and P. Zerwas, Heavy flavors at LEP I, preprint MPI-PAE/PTh 49/89, Max Planck Institute (Munich), a shortened version is available as J. Kühn and P. Zerwas in: Z Physics at LEP I, CERN 89-08, eds. G. Altarelli et al.(CERN, Geneva, 1989), Vol. 1, pp. 267-372.
[3] M. Böhm and W. Hollik, in: Z Physics at LEP I, CERN 89-08, eds. G. Altarelli et al. (CERN, Geneva, 1989), Vol. 1, pp. 203-234.

[4] MARK II Collab., J.F. Kral et al., Phys. Rev. Lett. 64 (1990) 1211.

[5] L3 Collab., B. Adeva et al., Phys. Lett. B 252 (1991) 713.

[6] OPAL Collab, M.Z. Akrawy et al., Phys. Lett. B 263 (1991) 311.

[7] ALEPH Collab., D. Decamp et al., Phys. Lett. B 263 (1991) 325.

[8] DELPHI Collab., P. Abreu et al., Phys. Lett. B 276 (1992) 536.

[9] L3 Collab., B. Adeva et al., Phys. Lett. B 288 (1992) 395.

[10] L3 Collab., B. Adeva et al., Nucl. Instrum. Methods A 289 (1990) 35.

[11] L3 Collab., B. Adeva et al., Phys. Lett. B 252 (1990) 703.

[12] T. Sjöstrand and M. Bengtsson, Comput. Phys. Commun. 43 (1987) 367;

T. Sjöstrand, in: Z Physics at LEP1, CERN-89-08, eds. G. Altarelli et al. (CERN, Geneva, 1989), Vol. 3, pp. 143-340,

for 1990 data we used JETSET 7.2 and for 1991 data we used JETSET 7.3.

[13] C. Peterson et al., Phys. Rev. D 27 (1983) 105.

[14] R. Brun et al., GEANT 3, CERN DD/EE/84-1 (revised) (September 1987).

[15] H. Fesefeldt, RWTH Aachen Report PITHA 85/02 (1985).

[16] L3 Collab., B. Adeva et al., Phys. Lett. B 261 (1991) 177.

[17] Particle Data Group, J.J. Hernández et al., Review of particle properties, Phys. Lett. B 239 (1990) VII.1 13.

[18] O. Adriani et al., Nucl. Instrum. Methods A 302 (1991) 53.

[19] D. Bardin et al., Z. Phys. C 44 (1989) 493; Nucl. Phys. B 351 (1991) 1; Phys. Lett. B 255 (1991) 290.

[20] L3 Collab., B. Adeva et al., Z. Phys. C 51 (1991) 179. 